\title{
Sleep Deprivation Affects Working Memory in Low but Not in High Complexity for the N-Back Test
}

\author{
Guadalupe J. Terán-Pérez ${ }^{1}$, Alejandra E. Ruiz-Contreras² ${ }^{2}$ Rosa O. González-Robles ${ }^{3}$, \\ Rosario Tarrago-Castellanos ${ }^{4}$, Roberto E. Mercadillo ${ }^{5}$, Anabel Jiménez-Anguiano ${ }^{4}$, \\ Javier Velázquez-Moctezuma ${ }^{4}$
}

${ }^{1}$ Sleep Disorder Laboratory, Universidad Autónoma Metropolitana-Iztapalapa, Iztapalapa, México; ${ }^{2}$ Laboratorio de Neurogenómica Cognitiva, Laboratorio de Psicofisiología, Facultad de Medicina, Universidad Nacional Autónoma de México, México City, México; ${ }^{3}$ Mathematic Department, Universidad Autónoma Metropolitana-Iztapalapa, Iztapalapa, México; ${ }^{4}$ Department of Reproductive Biology, Universidad Autónoma Metropolitana-Iztapalapa, Iztapalapa, México; ${ }^{5}$ Institute of Neurobiology, Universidad Nacional Autónoma de México, México City, México.

Email: gteran_s@hotmail.com,gjovannat@yahoo.com

Received October $24^{\text {th }}, 2012$; revised November $22^{\text {nd }}, 2012$; accepted November $30^{\text {th }}, 2012$

\begin{abstract}
Sleep clearly influences learning and memory since sleep deprivation and stress impairs both cognitive processes. Working memory is an essential cognitive process and refers to a short-term holding of incoming information required to update the long-term mnemonic storage and to manipulate new elements in order to solve problems and make decisions. Nevertheless, the influence of sleep deprivation on working memory has scarcely been studied. In this study we evaluated working memory using the N-back test after increasing periods of wakefulness. Healthy young males were kept awake for 36 hours and the two N-back tasks with low (1-Back) and high (3-Back) levels of complexity were applied every 6 hours. Additionally, salivary cortisol was determined along the study. Unlike the control non-deprived participants, the sleep deprived volunteers showed a significant decrease in their efficiency to solve the 1-Back task after 24 hours of sleep deprivation. However, no differences were observed after 30 and 36 hours of sleep deprivation. Concerning the 3-Back task no differences were observed after sleep deprivation. Regarding reaction time, the deprived group manifested slower responses for the 1-Back task and for the 3-Back task after 30 hours and 36 hours of sleep deprivation, respectively. Cortisol levels presented the normal daily oscillation and no differences were observed between groups. This data suggests that sleep deprivation affects basal states of attention instead of working memory while performing simple tasks. The impact of sleep deprivation on the cognitive performance depends on the moment of day when the task is applied and the complexity of the tests used to assess these mnemonic skills.
\end{abstract}

Keywords: Sleep Deprivation; Working Memory; Cortisol Levels; N-Back Task; Stress

\section{Introduction}

Sleep is an essential process for normal human development and daily cognitive performance [1,2]. Nowadays, however, the current urban life-style includes assorted and nocturnal schedules and activities that elicit stressful conditions as well as alterations in the sleep-wake rhythm $[3,4]$. Both stress and sleep deprivation have a negative impact on the satisfactory function of several cognitive processes, such as, attention, motor coordination, learning, language understanding and memory [5-9]. In pioneer observations, Jenkins and Dallenbach [10] reported the influence of sleep on memory consolidation since the ability to remember nonsense syllables is better after a

\footnotetext{
${ }^{*}$ No conflict of interest is declared.
}

sleep period than after a wakefulness period. Recently, studies have found that the lack of an adequate sleep period affects neural networks and behavioral manifestations involving the storage of novel experiences [11]. However, although working memory constitutes one of the main mnemonic processes it has scarcely been studied in relation to sleep disorders or disturbances. Working memory refers to a short-term reserve of incoming information required to update the long-term mnemonic storage and to manipulate new elements to solve problems and make decisions [12-16].

According to the Baddeley's model [13], the incoming information processed in working memory is controlled by a central executive system that receives, connects, and organizes visual and auditory perceptions to alternate 
among different tasks, such as orientation, inhibition of actions, and remembering. In addition, this system is coordinated through neural networks comprising the ventral and dorsal portions of the prefrontal cortex [13]. Both brain regions manifest intense activity during the performance of working memory tasks although neural activity in the dorsal portion is more evident when a subject displays a slower recovery of information [6]. Behavioral and neuroimaging studies suggest that the deterioration of working memory could be due to the effects of sleep deprivation on the functions of the prefrontal cortex [7,17]. The decreased metabolic signal observed in brain dynamics after sleep deprivation is related to the inhibition of cholinergic neurons located in the dorsal prefrontal cortex which is normally active during the rapid-eye-movement sleep stage [18].

Findings related to the effects of sleep deprivation on working memory are controversial. For example, sleep deprived individuals show an increase in reaction time when performing a verbal task either after 24 hours or 36 hours of deprivation, but the efficiency evaluated by the correct responses is not affected [14,19]. Reaction time also increases when individuals are restricted to only six hours of sleep a day for a week. In contrast with individuals sleeping eight hours daily, sleep restricted subjects exhibit an increase in reaction time while solving verbal and arithmetic working memory tasks [20-22].

Sleep deprivation implies a metabolic and cognitive extra-demand resulting in the activation of the hypothalamus-hypophysis-adrenal axis (HHA) [23]. In normal conditions, cortisol is released during early morning just before awakening. Sleep deprived individuals do not present high levels of cortisol during the morning, but do so in the afternoon between 18:00 and 23:00 hrs, inducing arousal and altering the normal sleep-wake rhythm $[24,25]$. Concerning memory, a poor execution of declarative memory tasks is clearly observed if a socially stressful condition is presented during the morning between 9:00 and 11:00 hrs. [26].

Moreover, correct responses and the reaction time decrease during the performance of N-Back tasks if it is executed immediately after a stressful condition [27]. NBack tests were designed to assess working memory skills since the processes required to solve the task involve the use and retention of incoming information through low (1-Back), medium (2-Back), or high (3-Back) levels of complexity [28-31].

The aim of this study was to assess the effect of increasing periods of sleep deprivation on working memory through the performance of n-Back tasks with different levels of complexity. We expected to find a decrease in the efficiency of working memory with longer sleep deprivation and more complex tasks.

\section{Method}

\subsection{Participants}

Eighteen right-handed and clinically healthy male students were recruited voluntarily from the Universidad Autónoma Metropolitana in Mexico City (age: $27 \pm 4$ years old). Neuropsychiatric and clinical interviews were applied to verify absence of sleep, medical and psychiatry disorders before the study. The Beta III test [32] was applied to select volunteers with a similar intellectual coefficient within the normal range of Mexican values. None of the participants was taking any medication, alcohol or illegal drugs during the study and none was paid for his participation. The experimental protocol was designed according to the principles offered by the Helsinki Declaration for medical research involving human subjects, and it was approved by the Bioethical Committee of the Universidad Autónoma Metropolitana. All the participants signed an informed consent before the study and after the nature of the experiment was explained.

\subsection{Experimental Procedure}

All volunteers were asked to maintain a seven hours daily sleeping program during two weeks before the study. Participants were randomly divided into two groups, control and deprived, each one made up of nine subjects who were kept at the sleep lab during a period of 48 hours. Participants in the control group were instructed to perform their normal nocturnal 7 - 8 hours' sleep period while participants in the deprived group kept awake during 36 hours. Since the study began at 08:00 participants were asked to solve two working memory tasks every six hours and saliva samples were taken at the same schedule to obtain a total of eight behavioral records and saliva samples: 08:00 (day 1, session one), 14:00 (day 1, session two), 20:00 (day 1, session three), 02:00 (day 2, session four), 08:00 (day 2, session five), 14:00 (day 2, session six), 20:00 (day 2, session seven), and 08:00 (day 3, session eight). Both control and deprived groups followed an equal protocol with the exemption of the 02:00 hrs sample on the second day when control participants were sleeping. Participants could talk, watch television, play videogames, read and do moderate physical exercise during the study if these activities did not represent stressful conditions. All the participants and activities were supervised by the research assistant. Similar light, noise, physical and social conditions were presented since the experiment was performed at the Sleep Disorder Laboratory of the Universidad Autónoma Metropolitana for all cases. All the participants were evaluated on the second night following a standard polysomnographic protocol to verify a normal brain and autonomic function during sleep. 


\subsection{Cognitive Tasks}

To assess working memory, two N-Back tests designed using E-prime: v 1.2 software (Psychology Software Tools, Inc., Pittsburgh, PA) were executed in a personal computer system. Briefly, the test was as follows: an $8 \times$ 8 matrix was presented in the center of the computer screen: horizontal visual angle (HVA): $4.30^{\circ}$; vertical visual angle (VVA): $3.60^{\circ}$. The matrix was depicted in dark gray on a light gray background to prevent the appearance of post-images. A dark gray-filled circle (HVA and VVA: $0.0315^{\circ}$ ) appeared during $500 \mathrm{~ms}$ in one of 60 of the 64 possible positions in the matrix; the four cells bordering the fixation point were not used during this task. For the 1-Back task participants were instructed to press one button whit their index finger if the position of the circle was the same as that presented one trial before and another button whit inverse index finger if it was different. For the 3-Back task participants were instructed to press one button whit their index finger if the position of the circle was the same as that presented three trials before and another button whit inverse index finger if it was different. A fixation cross in the center of the matrix (HVA: $0.6875^{\circ}$, VVA: $0.5730^{\circ}$ ) with $500 \mathrm{~ms}$ duration appeared between the trials throughout the experiment. Participants had $1500 \mathrm{~ms}$ to respond, timed from the moment when the circle appeared. A total of 120 stimuli were presented: 24 target trials and 96 non-target trials. The finger responses were collected by the E-prime software, which also provided the accuracy, omitted, correct and incorrect responses, and the reaction time for each stimulus. Participants performed two mock procedures at the beginning of the experiment to verify the proper understanding of the task. The detailed explanation of the task is presented in Figure 1.

\subsection{Cortisol Evaluation}

To assess salivary cortisol a saliva sample was taken immediately before the working memory task performance. Saliva was collected using a salivette (Sarstedt company) in the subjects' mouth during $30 \mathrm{~s}$. ELISA assay (Free Cortisol in Saliva ELISA, Inmuno Biological Laboratiroes IBL-America) was performed to measure the free cortisol levels [33,34]. All salivary samples were analyzed in duplicate. To determine the range of released cortisol levels the analysis was based on the normal distribution of cortisol in healthy people (User's Manual Free Cortisol in Saliva Elisa IBL). According to this normal distribution, a $1.8-14.7 \mathrm{ng} / \mathrm{ml}$ concentration of cortisol is presented immediately after awaking during the morning and decreases along the day displaying the lower range of $0.3-6 \mathrm{ng} / \mathrm{ml}$ during the afternoon.

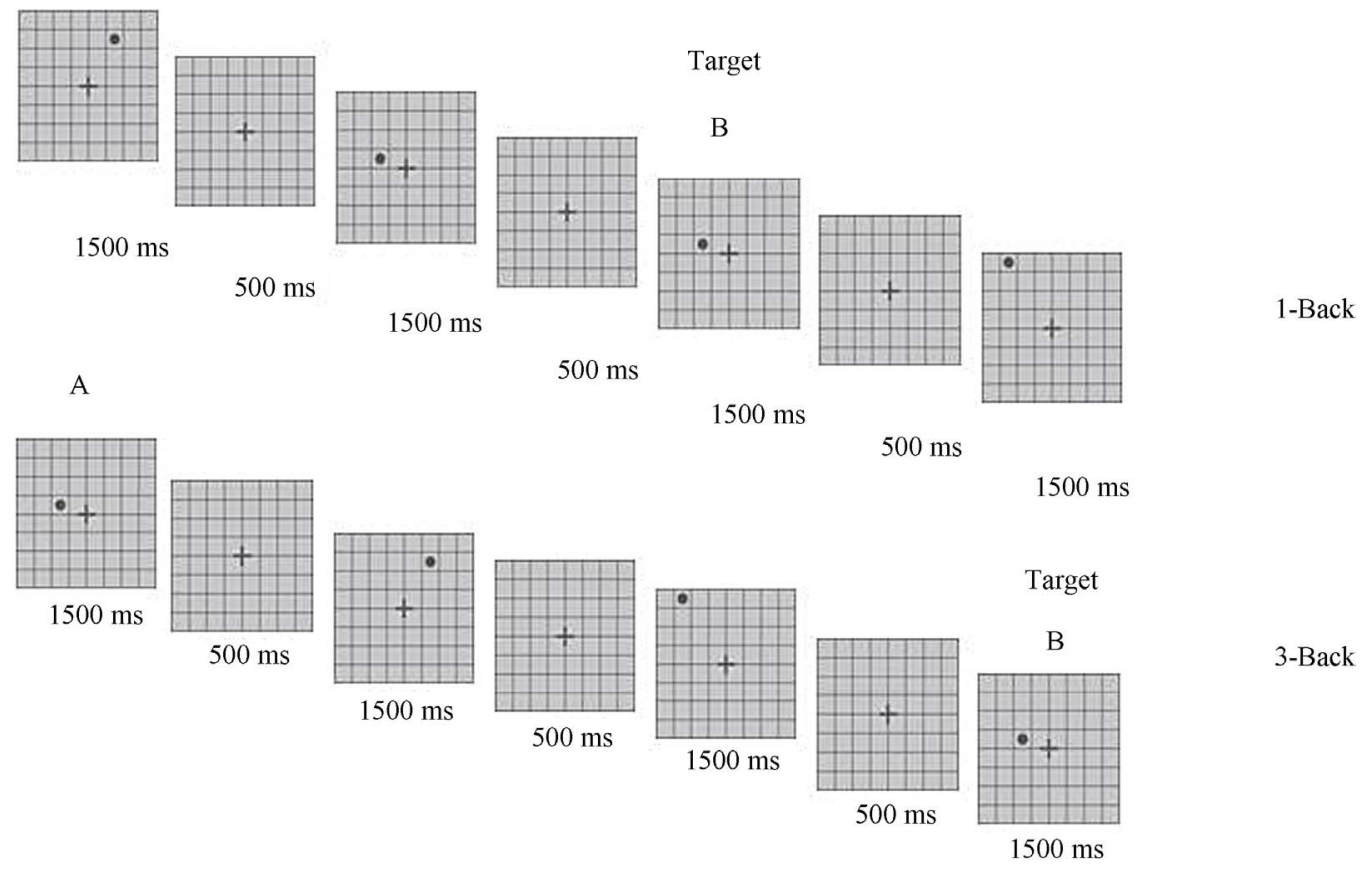

Figure 1. N-Back task. Matrix marked with letter B represents the position where the target stimulus (black circle) is presented. Matrix marked with letter A represents the trial-back that must be equal than the target stimulus. A simple matrix with no stimulus is presented between trials. Superior matrix illustrates the low complexity 1-Back task where the participant has to indicate with the movement of his index finger if the target stimulus $(B)$ is presented in the same position than the tral before (A). Inferior matrix illustrates the high complexity 3-Back task where the participant has to indicate with the movement of his index finger if the target stimulus (B) is presented in the same position than the three trials before (A). Each stimulus is projected during $500 \mathrm{~ms}$ and 64 possible positions in the matrix are available. 


\section{Statistical Analysis}

Database and statistical analyses were done in the SPSS 17 version software. An Adjusted Hit Rate (AHR) was calculated by the operation Hit Rate-minus-Error Rate (HR-ER) to assess the participants' efficiency in discriminating between target and non-target stimuli. HR was calculated dividing the number of correct responses for targets by the total number of target stimuli. ER was calculated dividing the number of errors for non-targets by the total number of non-target stimuli. An AHR $=0$ represents a response level equal to chance; all correct responses with no errors means an $\mathrm{AHR}=1$; all incorrect responses represents an AHR $=-1$ [35,31]. Mixed analysis of variance was calculated using Group (deprived $v s$ control) as the between groups factor (Sessions $1-8$ ), and Level of difficulty (1 vs. 3) as the within-subjects factor. To know the efficiency in task solving the mean of the AHR and the mean of the reaction time were used in the ANOVA analyses. To know the autonomic stress re- sponse the mean of cortisol levels in $\mathrm{ng} / \mathrm{ml}$ was used in the ANOVA analysis. To identify the distribution and changing rate of the working memory efficiency a learning curve was obtained by representing the mean of the AHR for each session in both the deprived and the control groups. Results with $p<0.05$ was considered as statistically significant.

\section{Results}

Data concerning AHR are displayed in Figure 2. When compared with the control group, the deprived group showed a significant decrease in the fifth session performed after 24 hours of continuous wakefulness $\left(F_{(1,16)}\right.$ $=5.85, p=0.03)$. No significant differences were observed in the following sessions.

Efficiency distributed in the learning curve indicated a lower proportion of correct responses for the 1-Back task after 24 hours of deprivation (session five) compared to session three before the sleep deprivation $\left(F_{(1,16)}=5.38\right.$,

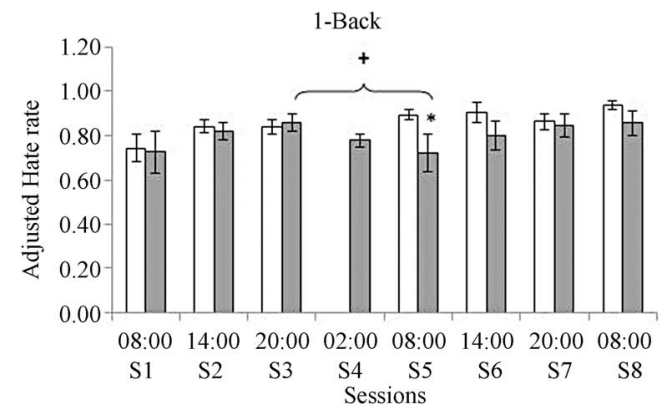

(a) $p=0.028)$ (see Figure 2).

Results concerning the reaction time indicated a slower execution in the deprived group for both the 1-Back and the 3-Back tasks. Significant differences were observed after 30 hours of deprivation in the 1-Back task $\left(F_{(1,16)}=\right.$ $7.40, p=0.01)$ and in the 3-Back task $\left(F_{(1,16)}=7.5, p=\right.$ 0.01 ) after 36 hours of deprivation (see Figure 3).

No differences between groups and within groups along the sessions were observed in the released levels of cortisol. Both deprived and control groups manifested higher levels of free cortisol released during the morning at session one, session five, and session eight (see Figure 4).

\section{Discussion and Conclusions}

According to previous reports [30,36,29], sleep deprivation or prolonged wakefulness results in a progressive impairment in the performance of tasks involving working memory and attention. Furthermore, the impairment should be progressive as deprivation increases. However, in the present study there was a significant decrease in the number of correct responses at 24 hours only and not after this time, although the reaction time for responses were always lower and the amount of incorrect responses was higher among the sleep deprived group in comparison to the control group. These results denote the importance of a full night's sleep to effectively resolve cognitive tasks requiring working memory processes $[19,29]$.

Moreover, cortisol has been repeatedly involved in memory process $[24,27,37,38]$. As no differences were observed in the present study between the sleep deprived and the control group concerning cortisol levels, the differences in the performance of both groups are not due to cortisol variations.

Both deprived and control groups manifested a normal oscillation of cortisol with higher levels during the morning $[24,38]$, which may be because 36 hours of sleep deprivation in artificial circumstances are not enough to

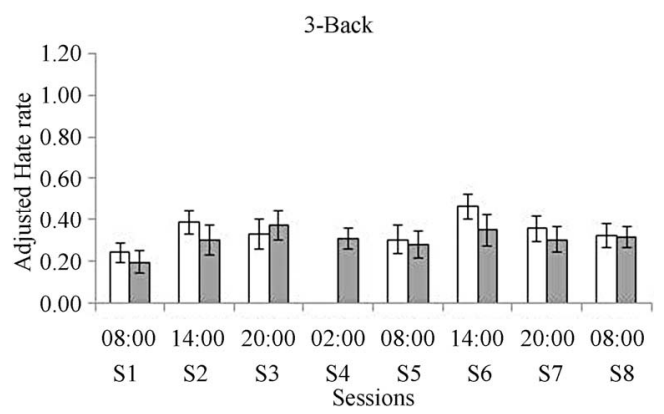

(b)

Figure 2. Comparison between groups for the Adjustment Hate Rate (AHR) obtained for the 1-Back task (a) and for the 3-Back task (b). White bars represent the control group and gray bars represent the deprived group. *Means significant differences between groups $p=\mathbf{0 . 0 3}$ when the ANOVA analysis was performed. +Represents differences within session 5 and session $3+p=0.01$. 


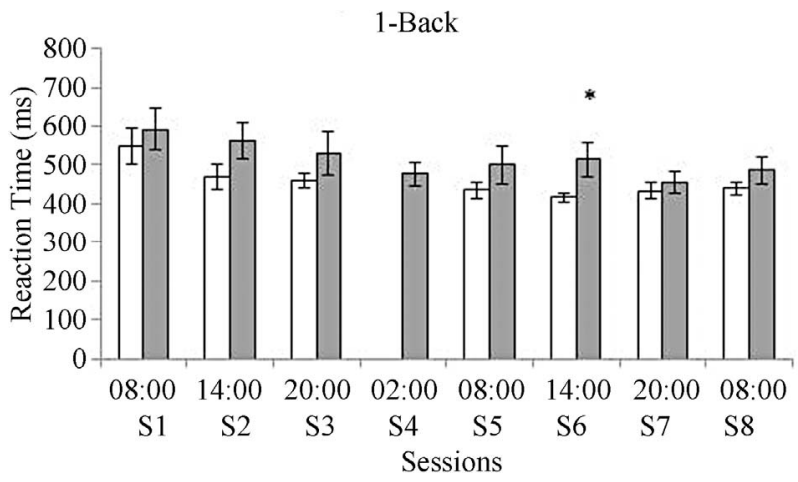

(a)

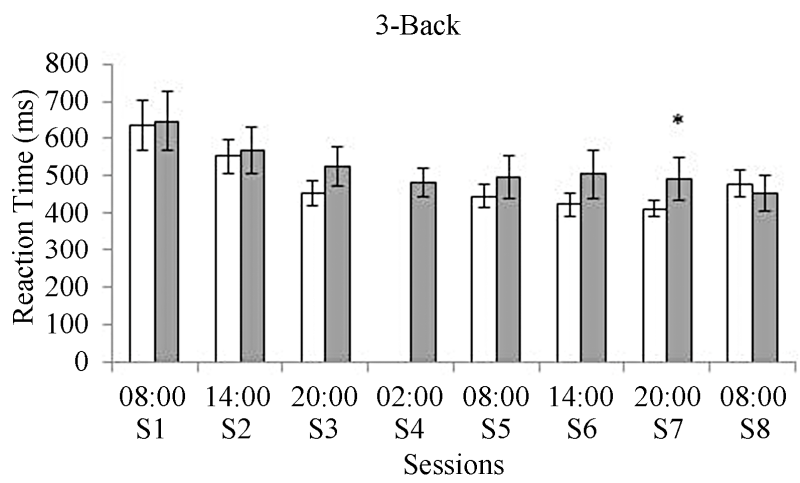

(b)

Figure 3. Reaction time for the 1-Back task (a) and for the 3-Back task (b). White bars represent the control group and gray bars represent the deprived group. *In graphic A means significant differences between groups in session six at $p=0.01$ when the ANOVA analysis was performed. *In graphic B means significant differences between groups in session seven at $p=0.01$ when the ANOVA analysis was performed.

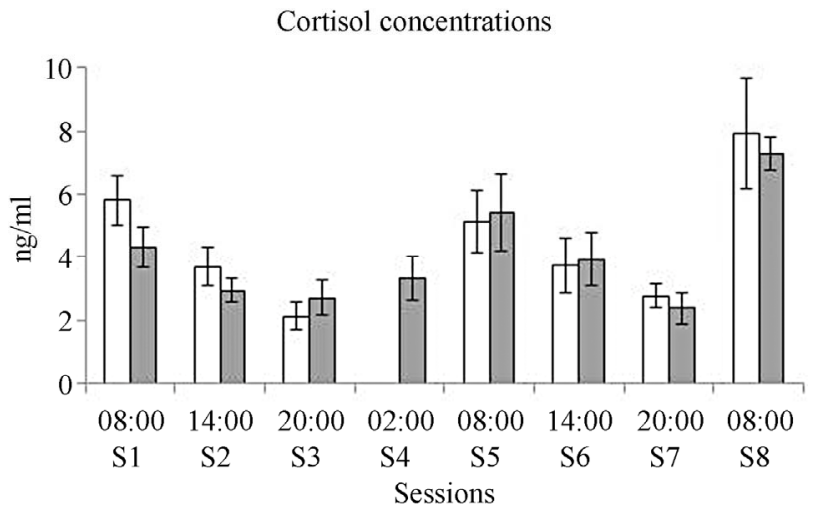

Figure 4. Mean for the levels of released cortisol along the sessions. White bars represent the control group and gray bars represent the deprived group.

elicit remarkable cortisol variations. These results are relevant since high levels of steroid hormones released by the adrenal cortex affect mnemonic processes and incorrect responses given to cognitive tasks could be related to the moment of the day when the task is performed [27,37]. For example, effectiveness decreases during the morning because of a high concentration of glucocorticoids and this concentration increases after sleep deprivation [26]. Therefore, the incorrect responses and the slower reaction time in the experimental group could be related to sleep deprivation but not to cortisol levels.

Concerning the N-back test it was expected that the more complex the task, the more difficulties would be presented in retaining, updating, and manipulating incoming information as it is required in working memory regulated by brain functions in the prefrontal cortex [26, $30,36]$. Nevertheless, only few significant differences were observed between groups in the simple task (1Back) while in the complex task (3-Back) no differences were observed in the number of correct responses or in the reaction time such as in other studies [26]. These results may imply that the prefrontal function required to solve a complex 3 -Back test is not affected by a 36 hours of sleep deprivation period or by stressful situations designed in experimental conditions, as used in this study. Performance for the simple 1-Back test was affected since these experimental conditions influence basal attention. Therefore, sleep deprivation in these experimental conditions may not affect working memory or executive processes regulated by ventral prefrontal and cingulate cortices [39], but affects attention manifested in a basal wake state regulated by a frontal-dorsal and parietal system for orientation [40]. Additionally, findings suggest that responses given for cognitive tasks do not necessarily correlate with reaction time and correct responses, so the model may evaluate basal attention and not purely working memory [9]. Participants invest more attention in a more complex cognitive task is and this is observed in normal or deprived situations [20].

Our results offer future directions for studying the influence of sleep deprivation on working memory. Concerning the N-back test, the results suggest that a more sensitive task must be developed for attention and memory, in order to properly assess the effects of prolonged wake and deprivation.

Acute sleep deprivation in artificial conditions does not elicit enough stress altering the adequate execution of working memory. The creation of a chronic experimental model may be needed to study theses effects.

Acute sleep deprivation may affect the basal level of attention in wake states to perform a simple working memory task but not the systems required in a more complex task.

\section{Acknowledgements}

We thank the Doctorado en Ciencias Biológicas y de la 
Salud program at the Universidad Autónoma Metropolitana to which I am currently enrolled, and has standard of excellence approved by CONACYT. And the financial support offered by CONACYT fellow number 28309. Grant IN217311 to ARRL. We also thank the members of the Sleep Disorder Laboratory at UAM-Iztapalapa and the volunteers who kindly agree to participate.

\section{REFERENCES}

[1] M. G. Frank, "The Mystery of Sleep Function: Current Perspectives and Future Directions," Reviews in the Neuroscience, Vol. 17, No. 4, 2006, pp. 375-392.

[2] E. Mignot, "Why We Sleep: The Temporal Organization of Recovery," PLoS Biology, Vol. 6, No. 4, 2008, pp. 661669.

[3] T. H. Monk, C. F. 3rd Reynolds, D. J. Buysse, J. M. DeGrazia and D. J. Kupfer, "The Relationship between Lifestyle Regularity and Subjective Sleep Quality," Chronobiology International, Vol. 20, No. 1, 2003, pp. 97-107.

[4] J. Waterhouse, Y. Fukuda and T. Morita, "Daily Rhythms of the Sleep-Wake Cycle," Journal of Physiology Anthropology, Vol. 31, No. 1, 2012, pp. 1-14.

[5] P. C. Kyllonen and R. E. Christal, "Reasoning Ability Is (Little More Than) Working Memory Capacity?!” Intelligence, Vol. 14, No. 4, 1990, pp. 389-433. doi:10.1016/S0160-2896(05)80012-1

[6] B. Rypma and M. D'Esposito, “The Roles of Prefrontal Brain Regions in Components of Working Memory: Effects of Memory Load and Individual Differences," Proceeding of National Academy of Sciences of the United States of America, Vol. 96, No. 11, 1999, pp. 6558-6663. doi:10.1073/pnas.96.11.6558

[7] W. C. Choo, W. W. Lee, V. Venkatraman, F. S. Sheu and M. W. Chee, "Dissociation of Cortical Regions Modulated Byboth Working Memory Load and Sleep Deprivation and by Sleep Deprivation Alone," Neuroimage, Vol. 25, No. 2, 2005, pp. 579-587.

doi:10.1016/j.neuroimage.2004.11.029

[8] J. Lim and D. F. Dinges, "Sleep Deprivation and Vigilant Attention," Annals of the New York Academy of Science, Vol. 1129, No. 1129, 2008, pp. 305-322. doi:10.1196/annals.1417.002

[9] N. Goel, H. Rao, J. S. Durmer and D. F. Dinges, "Neurocognitive Consequences of Sleep Deprivation," Seminars on Neurology, Vol. 29, No. 4, 2009, pp. 320-339. doi:10.1055/s-0029-1237117

[10] J. Jenkins and K. Dallenbach, "Obliviscence During Sleep and Waking," The American Journal of Psichology, Vol. 35, 1924, pp. 605-612. doi:10.2307/1414040

[11] S. S. Yoo, P. T. Hu, N. Gujar, F. A. Jolesz and M. P. Walker, "A Deficit in the Ability to Form New Human Memories without Sleep," Nature Neurosciene, Vol. 10, No. 3, 2007, pp. 385-392. doi:10.1038/nn1851

[12] A. Baddeley, "Working Memory," Science, Vol. 255, No. 5044, 1992, pp. 556-559. doi:10.1126/science.1736359
[13] A. Baddeley, "Working Memory," Current Biology, Vol. 20, No. 4, 2010, pp. 136-140. doi:10.1016/j.cub.2009.12.014

[14] M. W. Chee, L. Y. Chuah, V. Venkatraman, W. Y. Chan, P. Philip and D. F. Dinges, "Functional Imaging of Working Memory Following Normal Sleep and after 24 and 35 $\mathrm{h}$ of Sleep Deprivation: Correlations of Fronto- parietal Activation with Performance," Neuroimage, Vol. 31, No. 1, 2006, pp. 419-428. doi:10.1016/j.neuroimage.2005.12.001

[15] E. K. Vogel, G. F. Woodman and S. J. Luck, "Storage of Features, Conjunctions and Objects in Visual Working Memory," Journal of Experimental Psychology, Human Perception and Performance, Vol. 27, No. 1, 2001, pp. 92-114.

[16] T. H. Turner, S. P. Drummond, J. S. Salamat and G. G. Brown, "Effects of $42 \mathrm{Hr}$ of Total Sleep Deprivation on Component Processes of Verbal Working Memory," Neuropsychology, Vol. 21, No. 6, 2007, pp. 787-795. doi:10.1037/0894-4105.21.6.787

[17] K. Jones and Y. Harrison, "Frontal Lobe Function, Sleep Loss and Fragmented Sleep," Sleep Medicine Reviews, Vol. 5, No. 6, 2001, pp. 463-475. doi:10.1053/smrv.2001.0203

[18] A. Muzur, E. F. Pace-Schott and J. A. Hobson, "The Prefrontal Cortex in Sleep," Trends in Cognitive Science, Vol. 6, No. 11, 2002, pp. 475-481. doi:10.1016/S1364-6613(02)01992-7

[19] P. A. Reuter-Lorenz, J. Jonides, E. E. Smith, A. Hartley, A. Miller, C. Marshuetz and R. A. Koeppe, "Age Differences in the Frontal Lateralization of Verbal and Spatial Working Memory Revealed by PET," Journal of Cognitive Neuroscience, Vol. 12, No. 1, 2000, pp. 174-187. doi:10.1162/089892900561814

[20] M. W. Chee and W. C. Choo, "Functional Imaging of Working Memory after $24 \mathrm{Hr}$. of Total Sleep Deprivation," Journal of Neuroscience, Vol. 24, No. 19, 2004, pp. 4560-4567. doi:10.1523/JNEUROSCI.0007-04.2004

[21] S. P. Drummond, G. G. Brown, J. C. Gillin, J. L. Stricker, E. C. Wong and R. B. Buxton, "Altered Brain Response to Verbal Learning Following Sleep Deprivation," Nature, Vol. 403, No. 6770, 2000, pp. 655-657. doi: $10.1038 / 35001068$

[22] F. Jiang, R. D. VanDyke, J. Zhang, F. Li, D. Gozal and X. Shen, "Effect of Chronic Sleep Restriction on Sleepiness and Working Memory in Adolescents and Young Adu1ts," Journal of Clinical and Experimental Neuropsychology, Vol. 33, No. 8, 2011, pp. 892-900. doi:10.1080/13803395.2011.570252

[23] B. S. McEwen, "Sleep Deprivation as a Neurobiologic and Physiologic Stressor: Allostasis and Allostatic Load," Metabolism, Vol. 55, No. 10, 2006, pp. S20-S23. doi:10.1016/j.metabol.2006.07.008

[24] R. Leproult, G. Copinschi, O. Buxton and E. Van Cauter, "Sleep Loss Results in an Elevation of Cortisol Levels the Next Evening," Sleep, Vol. 20, No. 10, 1997, pp. 865-870.

[25] A. Omisade, O. M. Buxton and B. Rusak, "Impact of Acute Sleep Restriction on Cortisol and Leptin Levels in 
Young Women," Physiological Behavior, Vol. 99, No. 5, 2011, pp. 651-656. doi:10.1016/j.physbeh.2010.01.028

[26] F. S. Maheu, P. Collicutt, R. Kornik, R. Moszkowski and S. J. Lupien, "The Perfect Time to be Stressed: A Differential Modulation of Human Memory by Stress Applied in the Morning or in the Afternoon," Progress in Neuropsychopharmacology and Biological Psychiatry, Vol. 29, No. 8, 2005, pp. 1281-1288.

[27] D. Schoofs, D. Preub and O. Wolf, "Psychosocial Stress Induces Working Memory Impairments in an N-Back Paradigm," Psychoneuroendocrinology, Vol. 33, No. 5, 2008, pp. 643-653. doi:10.1016/j.psyneuen.2008.02.004

[28] J. H. Callicott, V. S. Mattay, A. Bertolino, K. Finn, R. Coppola, J. A. Frank, T. E. Goldberg and D. R. Weinberger, "Physiological Characteristics of Capacity Constraints in Working Memory as Revealed by Functional MRI," Cerebral Cortex, Vol. 9, No. 1, 1999, pp. 20-26. doi:10.1016/j.psyneuen.2008.02.004

[29] K. Kuriyama, K. Mishima H. Suzuki, S. Aritake and M. Uchiyama, "Sleep Accelerates the Improvement in Working Memory Performance," Journal of Neuroscience, Vol. 28, No. 40, 2008, pp. 10145-10150. doi:10.1523/JNEUROSCI.2039-08.2008

[30] A. M. Owen, K. M. McMillan, A. R. Laird and E. Bullmore, "N-Back Working Memory Paradigm: A MetaAnalysis of Normative Functional Neuroimaging Studies," Hum Brain Mapping, Vol. 25, No. 1, 2005, pp. 4659. doi:10.1002/hbm.20131

[31] A. E. Ruiz-Contreras, G. Soria-Rodríguez, G. A. Almeida-Rosas, P. A. García-Vaca, M. Delgado-Herrera, M. Méndez-Díaz and O. Prospéro-García, "Low Diversity and Low Frequency of Participation in Leisure Activities Compromise Working Memoryefficiency in Young Adu1ts," Acta Psychologica, Vol. 139, No. 1, 2012, pp. 91-96. doi:10.1016/i.actpsy.2011.10.011

[32] C. E. Kellog and N. W. Morton, "Beta III," Manual Moderno, México, 2003.

[33] D. H. Hellhammer, S. Wüst and B. M. Kudielka, "Sali- vary Cortisol as a Biomarker in Stress Research," Psychoneuroendocrinology, Vol. 34, No. 2, 2009, pp. 163-171.

[34] W. J. Inder, G. Dimeski and A. Russell, "Measurement of Salivary Cortisol in 2012-Laboratory Techniques and Clinical Indications," Clinical Endocrinology, Vol. 77, No. 5, 2012, pp. 645-651. doi:10.1111/j.1365-2265.2012.04508.x

[35] A. Abi-Dargham, O. Mawlawi, I. Lombardo, R. Gil, D. Martinez, Y. Huang, D. R. Hwang, J. Keilp, L. Kochan, R. Van Heertum, J. M. Gorman and M. Laruelle, "Prefrontal Dopamine D1 Receptors and Working Memory in Schizophrenia," Journal of Neuroscience, Vol. 22, No. 9, 2002, pp. 3708-3719.

[36] T. S. Braver, J. D. Cohen, L. E. Nystrom, J. Jonides, E. E. Smith and D. C. Noll, "A Parametric Study of Prefrontal Cortex Involvement in Human Working Memory," Neuroimage, Vol. 5, No. 1, 1997, pp. 49-62. doi:10.1006/nimg.1996.0247

[37] E. R. De Kloet, M. S. Oitzl and M. Joëls, "Stress and Cognition: Are Corticosteroids Good or Bad Guys?" Trends in Neuroscience, Vol. 22, No. 10, 1999, pp. 422-426. doi:10.1016/S0166-2236(99)01438-1

[38] K. Dedovic, A. Duchesne, J. Andrews, V. Engert and J. C. Pruessner, "The Brain and the Stress Axis: The Neural Correlates of Cortisol Regulation in Response to Stress," Neuroimage, Vol. 47, No. 3, 2009, pp. 864-871. doi:10.1016/j.neuroimage.2009.05.074

[39] C. Libedinsky, D. V. Smith, C. S. Teng, P. Namburi, V. W. Chen, S. A. Huettel and M. W. Chee, "Sleep Deprivation Alters Valuation Signals in the Ventromedial Prefrontal Cortex," Frontiers in Behavioral Neuroscience, Vol. 5, No. 1-10, 2011, p. 70. doi: $10.3389 /$ fnbeh.2011.00070

[40] S. E. Petersen and M. I. Posner, "The Attention System of the Human Brain: 20 Years after," Annual Review of Neuroscience, Vol. 35, No. 35, 2012, pp. 73-89. doi:10.1146/annurev-neuro-062111-150525 